TITLE NUCLEAR EFFECTS ON HEAVY QUARK PRODUCTION, RESULTS FROM FERMILAB EXPERIMENTS E772 AND E789

Authof,s): M. 1. Leitch, D. Alde, H. Baer, J. Boissevain, T. Carey, G. T. Garvey, R. Jeppesen, J. Kapustinsky, A. Kle in, D. Lane, C. Lee, J. Liliberg. P. McGaughey, J. M. Moss, J. C. Peng, M. Brooks, G. Brown, D. Isenhower, M. Sadler, R. Schnathorst, G. Danner, M. Wang, L. Lederman, M. Schub, C. N. Brown, W. E. Cooper, H. Glass, Y. B. Hsiung, C. S. Mishra, K. Gounder, M. R. Adams, G. Gidal, P.-M. Ho, M. Kowitt, K.-B. Luk, D. Pripstein, M. Apol inski, R. Guo, D. M. Kaplan, V. Martin, R. Preston, J. ja, V. Tantkella, R. Childers, C. Darden, J. Wilson, R. L. McCarthy, Y.-C. Cher, G.-C. Kiang, P.-K. Teng, M. Bart.lett, and G. Hot lann.

SIJBMITTED TO: Quark Matter 199l, Gat1 inbury, Tennessee, November 11-15, 199l

$$
\text { in....ivith }
$$




\section{Nuclear Effects on Heavy Quark Production Results from Fermilab Experiments E772 and E789}

M. J. Leitch, D. Alde, H. Baer, J. Boissevain, T. Carey, G. T. Garvey, R. Jeppesen, J. Kapustinsky, A. Klein, D. Lane, C. Lee,

J. Lillberg, P. McGaughey, J. M. Moss, J. C. Peng Los Alamos National Laboratory, Los Alamos, New Mexico

M. Brooks, G. Brown, D. Lsenhower, M. Sadler, R. Schnathorst, Abilene Christian University, Abilent, Texas

G. Danner, M. Wang. Cisse Western Reserve, Clevel.nnd, Ohio

L. Lederman. M. Schub Univer:sity of Chicago, Chicago, Illinois

C. N. Brown, W. E. Cooper, H. Glass, Y. B. Elsiung, C. S. Mishra, K. Gounder Fermilab, Batavia, [llinoi:s M. R. Adami University of (llinois at Chicagn, Chicago, Illinolis G. Gidal, P.-M. Ho, M. Kowitt, K.-B. Luk, D. Pripstein Lawrence Berkeley Laboratory, Berkeley, California

M. Apolinski, R. Gun, D. M. Kaplan, V. Martin,

R. Preston. J. Sa, V. Tanikella Northern Illinois University, DeKalb, Illinoi:i

R. C'hilder:s, C. Darden, J. Wilison University of South Carolina, Columbia. South Carolina

R. L. Mi:Carthy, SifN? at Stonny Brook, Stony Frook. Now York

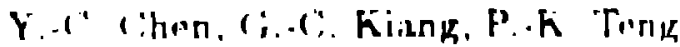

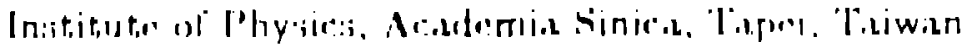
$.2 n \cdot 1$

if H.ur!.loti, 1: Holim,tun.

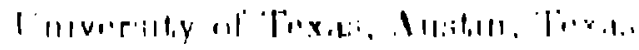

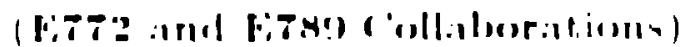




\section{Introduction}

Furinilab Experiments E772 and E789 are fixed target experiments with $800 \mathrm{GeV}$ protons incident on nuclear targets corresponding to a center-of-mass energy of $\sqrt{s} \sim$ $39 \mathrm{GeV}$. Measurements are made with a pair spectrometer which has a solid angle of a few percent and operates at high luminosity with up to $\sim 10^{12}$ (E772) or $\sim 10^{11}$ (E789) protons/spill. Our experimental program explores several types of nuclear medium effects: the modification of quark and gluon structure functions by the nucleus, effects on the production of vector mesons (e.g. $J / \psi$ and $\Upsilon$ ), and effects on the production of $\mathrm{D}$ mesons. The latter is accomplished with the use of a new silicon vertex detector. E789 also looks at the decays of $B$ mesons including the decay to $J / \psi$ and searches for the decays to two-chargerl particles (e.g. $B \rightarrow h^{+} h^{-}$) but I will not discuss this part of our program in this talk.

II. Structure Functions

The dominant procens:es involved in Urell-Yan (DY) and in vector meson production are shown in Fig. 1. The:se procentise:s provide information on the structure functions of the struck partons, which give the probability tor the parton involved to have a certain frartion, $x$, of the nuclenn momentum. The DY process involves the annihilation of a proton beam quark (anti-quark) with a target anti-quark (quark). Unlike deep inelastic lepton scattering where one is sensitive to both the target quark and anti-quark structure functions DY is primarily sensitive to the target anti-quark structure function in the kinematic region of our experiment. For resonance production $\left(J / \psi, \psi^{\prime}\right.$, and $\left.T\right)$ the dominant meethanism is that of gluon-gluon fusion which involves the product of the beam and target structure: finctions.

The busic: physir:al idtelas toehind a number of the common theoretical modelis tor the moditication of structure: tunctions by the nucleus can be illustrated in fairly simpie terms. In the discussion that follows I will look at the ratio of structure function:s between a heavy nucleus and deuterium, $R(A / D)$, versus target quark momentum fraction, $I_{2}$. A constant vilue of one corresponds to no nuclear effect. $I_{1}\left(I_{2}\right)$ represents the beam(target) parton momentum fraction and $I_{F}=I_{1}-I_{2}$.

i) Nuclear binding and fermi motion ${ }^{\text {-3 }}$ :

In principle nuclear binding will produce excess pions in the nucleus which would carey off some of the nut. lone: momentium and thus cause a reduction in the apparent, momentum fraction of the 'ilusks in the nus:enn. This produces an effer.t in the ratio as shown in Fig. 2a. How wever the pion has a valence anti-quark (which has a fairly hard momentum friction) while: the nucleosn has only soft (sea) anti-quark. Thuss the excess pions in a heavy nucle:us might be: exper:ted to cause an increase of the: ration at large $I$ for the [Dreli-Yin prores:s: which iis primarily sensitive to the anti-quarks (F'ig. 2b).

At large 2 the nuc:leson istructiure tiunction beromes pelatively amall. Hlowever formi

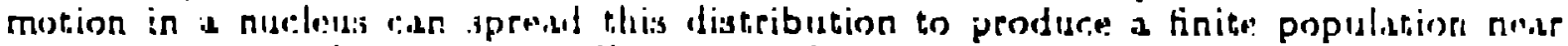

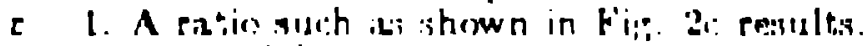

ii) Resiraling "i:

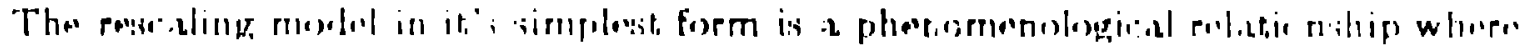

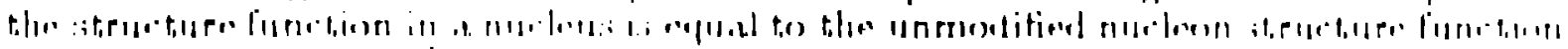

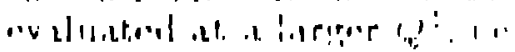

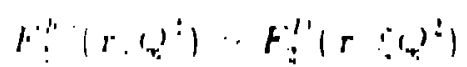

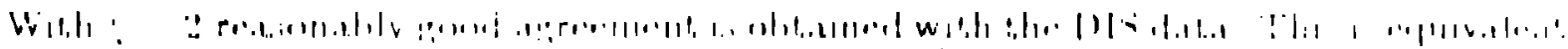

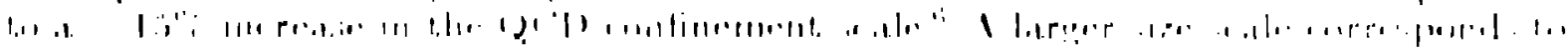

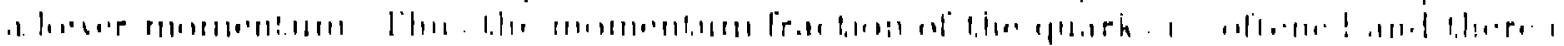


a loss of valence quark momentum. The resulting dependence of the ratio will then look something like Fig. 2d. One simple mociel which produces such an increase of scale is that of Close, Jaffee, Roberts, and Ross ${ }^{6}$ where they intruduce an increased confinement scale that is proportional to the overlap of nucleons. The nucleon overlap is calculated using different correlation functions and single-particle deusities. They then assume that when two nucleons overlap their quarks prcpagate over a larger spatial domain resulting in a corresponding increase in scale.
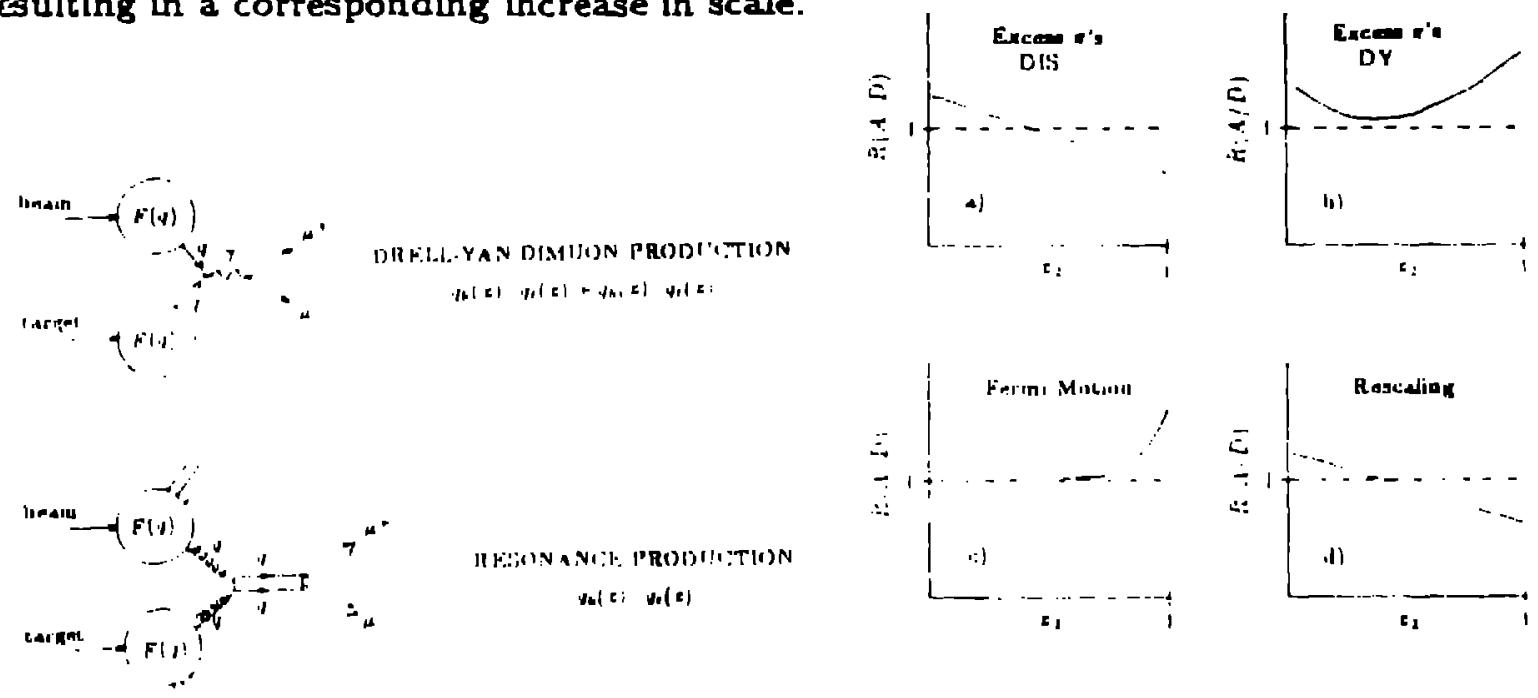

Fig. 1. The Deell-Yan and gluon-gluon fusion proressess.
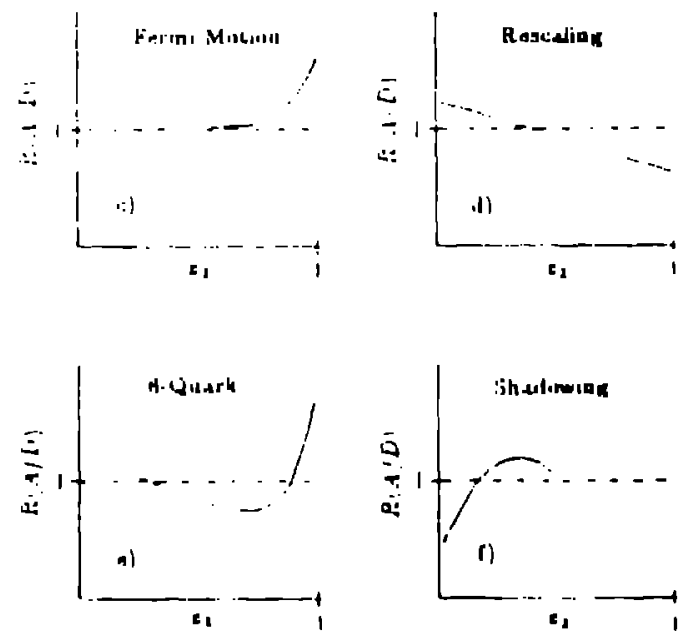

Fig. 2. Schematic: expectasuions for different E'MIC: moxtels as labeleat.

iii) Multi-qu ark clustersi't:

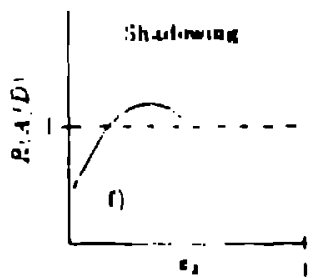

Multi-qua-k cluiter models obtain a larger length scale by assuming a probability $(-16-30 \%)$ for nurleons in a nur.leus to form six or more quark clusters. This should produce the same kind of (dependence on $x$ in the ratios as does rescaling. Howrver, as shown in Fig. 2e, a relative enhinesement near $x$ l should also be produced since nne quark can asyume the momentium of a single: nuclenn or more.

iv) : hadowing:

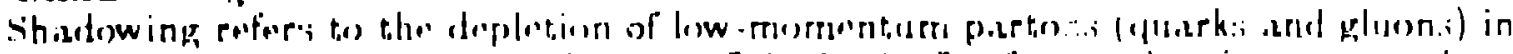

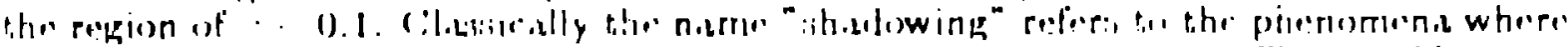

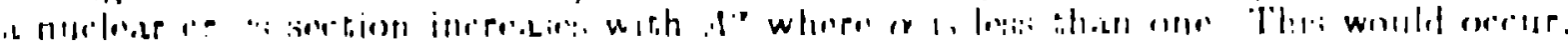

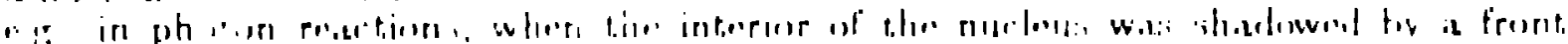

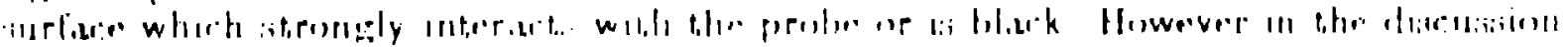

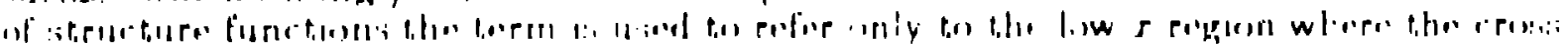

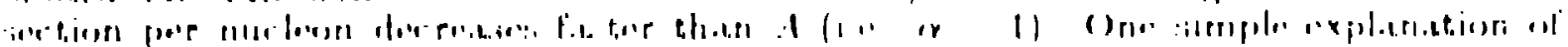

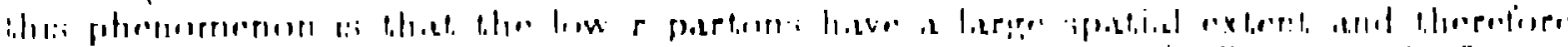

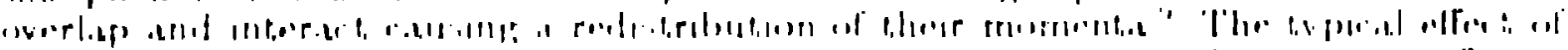

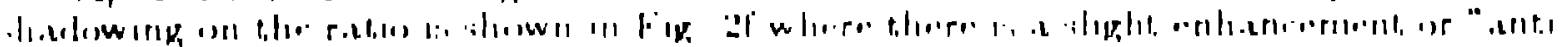

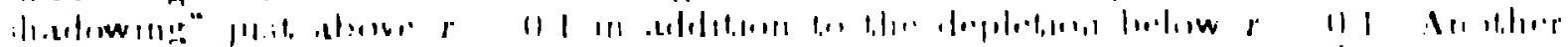

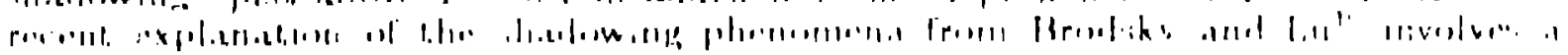

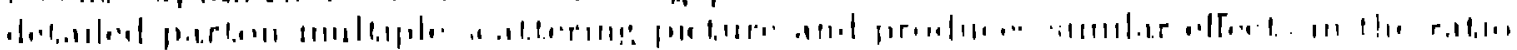




\section{Reaction Dyramies}

A number of reaction dramics effect must be considered when studying these hirg-energy reactions. 11 Some of these are depicted in cartoon form in Fig. 3. For the Drelf-Yen process we expect to see some initial-state multiple seattering and eners loss effects which will brosden the transverse momentum, PT, of the dimuon pair and shift the effective $x_{r}$ of the interaction. In the case of resonance production, e.g. $J / \psi$ production, other final-state effec's enter and the situation is more complicated. $\mathbf{A} c \bar{c}$ or pre $J / \psi$ peir iv formed in an almost point-like interaction and then must spread out for a considerable distance (e.g. 5-10 nuclear radii) at which it achieves a separation distance corresponding to the $J / \psi$ diameter and it hadronizes. The actual distance of course depends on both the $x \boldsymbol{F}$ at which it is pridised and differs according to what meson is produced. In addition a splash of low-energy $\pi$ 's, $\rho$ 's, and nur.leons is created by the incident beam quarks. Some of these can be ro-moving with the pre- $J / \psi$, i.e. have small relative veloxity with respert to the pre-J/ $\downarrow$. The pre-Jiw can then be multiple scattered or can be disson:iated by the co-movers, e.g. $J / \psi+i \rightarrow C+\bar{D}+\boldsymbol{X}$. The pre-J/ $\psi$ can also be dissociated by the nuclear medium directly, however since the co-movers can continue to interact well outside of the nuelear volume they may in principle have a mure important effect.

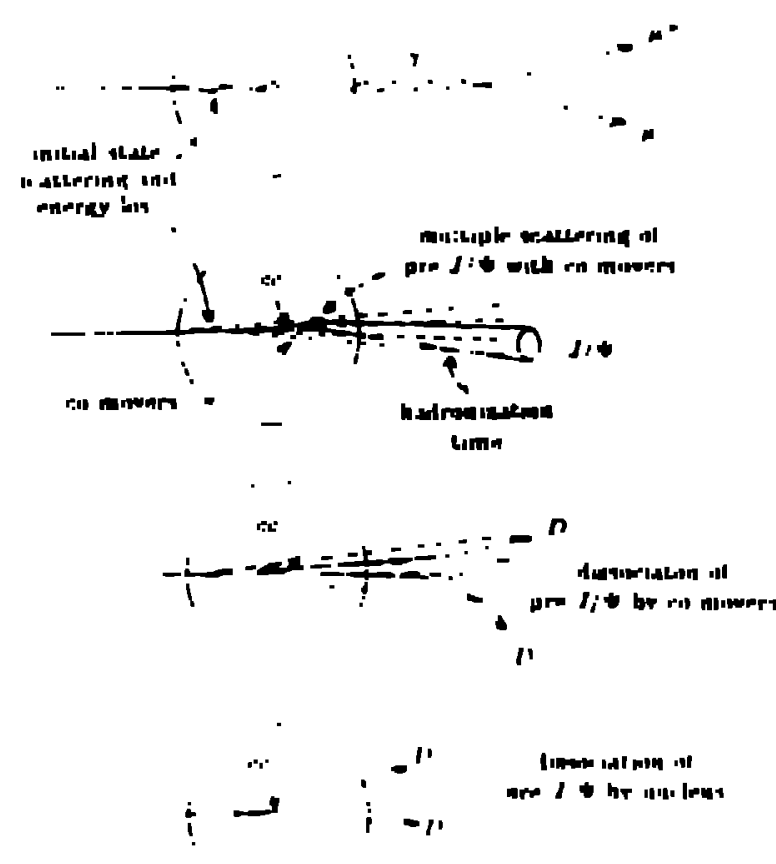

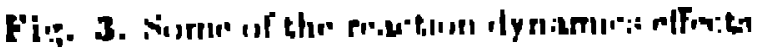

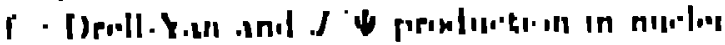
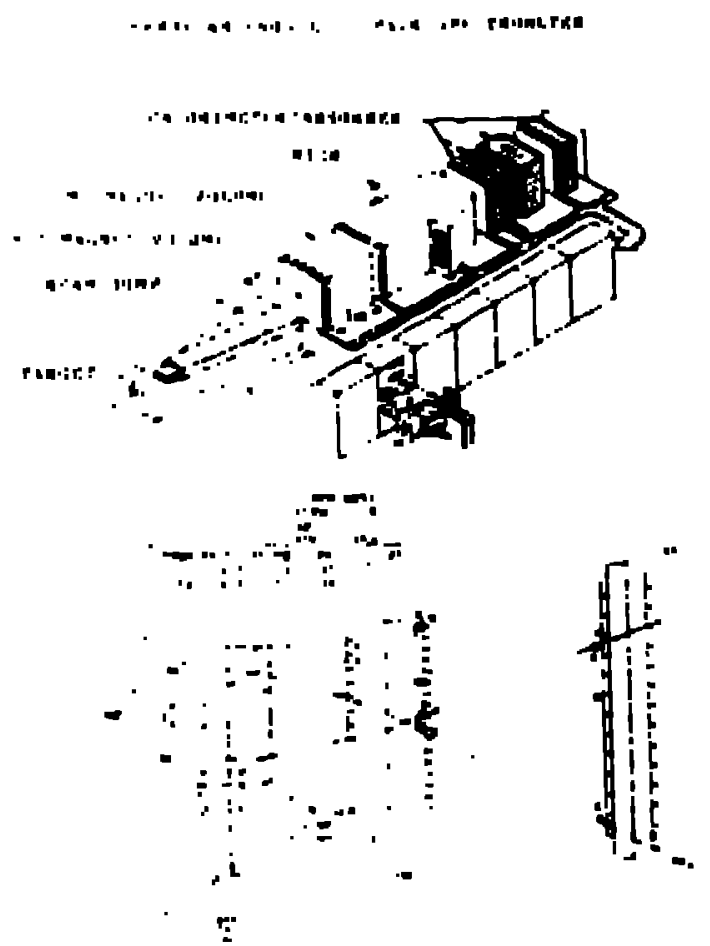

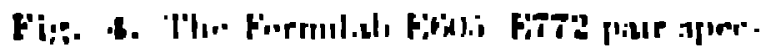

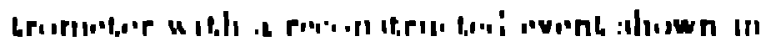

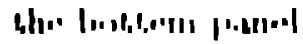

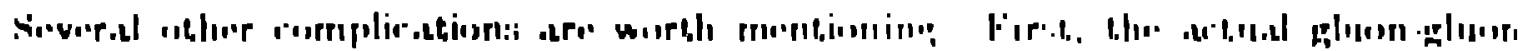

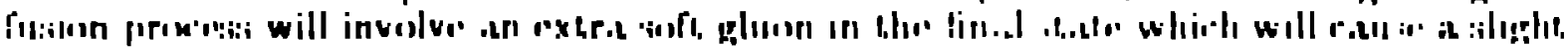

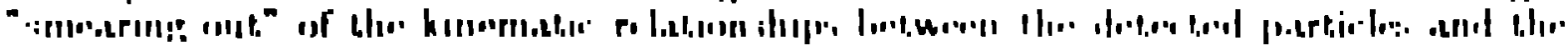

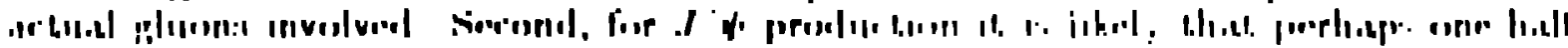

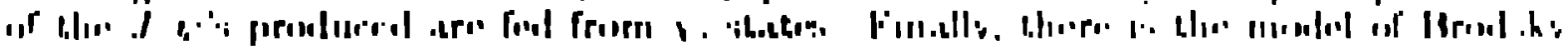

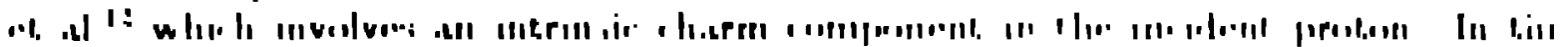

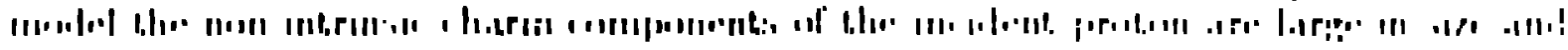


are stripped off on the black nuclear surface according to $A^{2 / 3}$. This leaves a spatially small $e \bar{c}$ which can then readily penetrate the nucleus and become a $J / \psi$. An analogous process involving intrinsic beauty could cnntribute to $\mathbf{Y}$ production. These procenses could then cause an $A^{2 / 3}$ dependence in the ratio at large $I_{f}$ where they would be expected to provide the docinant contribution to the cross section.

IV. Experiment ETt $2^{13}$

Fermilab E772 measures the A-dependence of the Drelt-Yan proceas and of resonance production at a $=-\mathrm{m}$. energy of $\sim 39 \mathrm{GeV}$ by colliding $800 \mathrm{GeV}$ protons with fixed nuclear targets. By measuring the momenta of the muon pair on:a can then calculate the beam parton and target parton momentum fractions. For the DY process the differential cross section is related to the structure functions by,

$$
\frac{d^{\prime} \sigma}{d: W d I}=K^{-}\left(Q^{2}, x_{F}\right) \frac{8 \pi \alpha^{2}}{9 M^{3}} \frac{x_{1} x_{2}}{x_{1}+x_{2}} \sum e_{1}^{2}\left\{F_{1}^{1}\left(x_{1}\right) \bar{F}_{1}^{\prime 2}\left(I_{2}\right) \div \bar{F}_{1}^{1}\left(x_{1}\right) F_{1}^{2}\left(x_{2}\right)\right\} .
$$

where $I_{1}\left(\Sigma_{2}\right)$ is the beam (target) quark momentum fraction and $F_{1}^{J}\left(\bar{F}_{i}^{J}\right)$ is the quark (anti-quark) strt:cture function with $j=1,2$ corresponding to beam, target, and where the sum over $i$ is over types of quarks. The $K$-factor in front of this expression represents QCD corrections and is $\sim 2$; however the cross-section factorizes ${ }^{14}$ so that this factor is constant over the quark momentum fraction. Thus in our experiment, which for the DY process involves almost exclusively the product of the beam quark and target anti-quark structure functions, the cross sertion is a direct measurement of this structure function product.

The detect.: used at Fermilab for E772 is shown in Fig. 1. Drell-Yan cross sections in the $3 \leq M_{\mu+\mu}-\leq 16 G c V$ region are only a few $p b^{\prime} s / G e b$. However this detector has good acceptance for $\mu^{+} \mu^{-}$pairs $(\simeq 5 \%)$ and can handle a very high rate of protons on target (up to $2 \times 10^{12} /$ spill). The portion of the $800 \mathrm{GeV}$ protons which does not interact in the target is absorbed in the beam dump contained within the first magnet. Produced muon pairs are analyzed by two large magnets with a total pTkick of abou: $6 \mathrm{GeV}$. A copper; carbon/polyethylene absorber near the rear of the first magnet protects the detectors downstream of the first magnet from low energy particles from the target, beam dump. or magnet walls. Several seti of scintillatur hodoscopes and wire chamber or drift chamber planes throughout the rest of the detector arcurately determine the momenta of the two muons. A calorimeter and a thick absorber assure that only muons penetrate through to the rearmost planes of deter:tors which then provide a clear muon identification. The measured momenta and the track angles are then used to reconstruct physirs quantities. The resulting tnass spectrum is shown in Fig. 5. In addition to the Des!l-Yan continisum we also seon the $J{ }^{\prime} \psi$ and $\psi^{\prime}$ peaks: near the lower end of the arrepotance and several $T$ peaks near the high end. For thi: Drell-Yan results that will be shown throughout the rest, of thi:i paper only the cross-

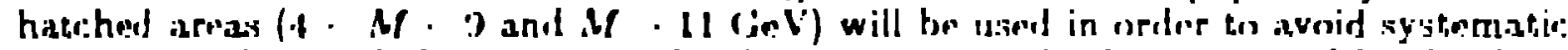

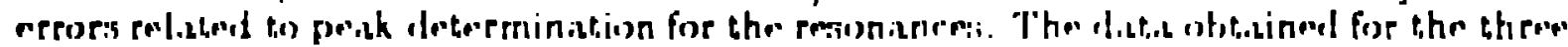

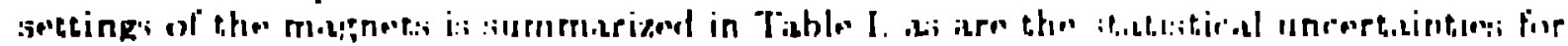

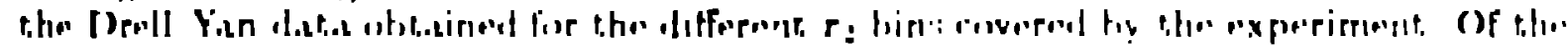

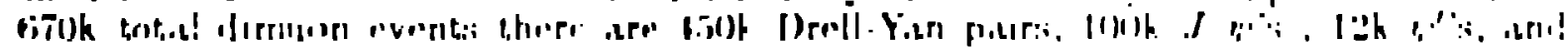

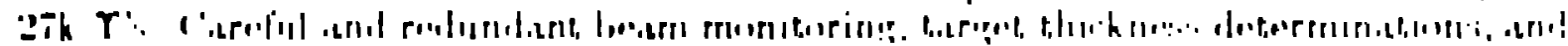

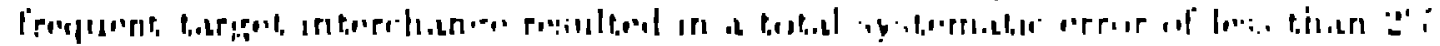

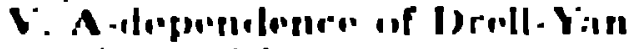

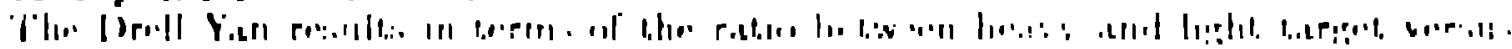

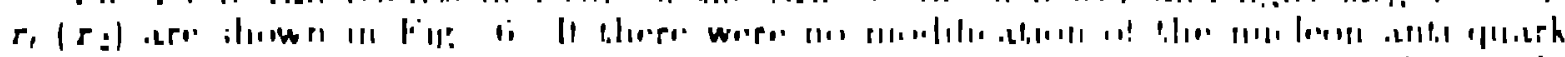

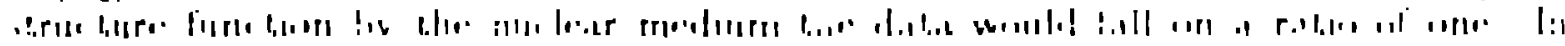

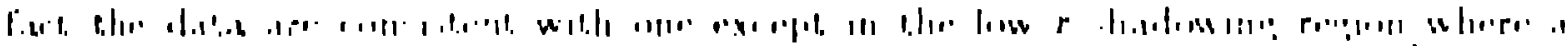

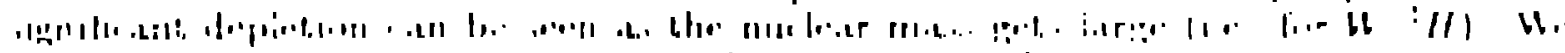

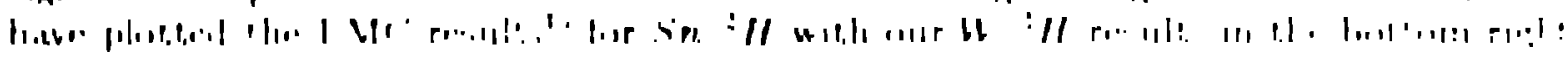


TABLE I - ET72 DATA SUMMARY

\begin{tabular}{lcccc}
\hline \hline Magnet Setting & $\left\langle M\left(\mu^{+} \mu^{-}\right)\right\rangle$ & Targets & Proton Flux & Dimuon Events \\
\hline Low mass & $4.8 \mathrm{GeV}$ & $F e / C a / L D_{2}$ & $6 \times 10^{15}$ & $170 \mathrm{k}$ \\
& & $W / C / L D_{2}$ & $2 \times 10^{15}$ & $70 \mathrm{k}$ \\
Medium mass & $6.5 \mathrm{GeV}$ & $F e / C a / L D_{2}$ & $2.4 \times 10^{16}$ & $280 \mathrm{k}$ \\
& & $W / C / L D_{2}$ & $0.9 \times 10^{16}$ & $110 \mathrm{k}$ \\
I.igh mass & $9.1 \mathrm{GeV}$ & $F e / C a / L D_{2}$ & $1.5 \times 10^{16}$ & $40 \mathrm{k}$ \\
& & & $5.6 \times 10^{16}$ & $670 \mathrm{k}$ \\
\hline
\end{tabular}

Statistical Errors in. Drell-Yan Cross Section Ratios for Various $x_{2}$ Bins $\left(x_{r}>0\right)$

\begin{tabular}{lcccccc}
\hline Target & $0-0.05$ & $0.05-0.1$ & $0.1-0.15$ & $0.15-0.2$ & $0.2-0.25$ & $0.25-0.3$ \\
\hline C2, Fe & $1 \%$ & $<1 \%$ & $1 \%$ & $2 \%$ & $5 \%$ & $12 \%$ \\
$C, W$ & $2 \%$ & $1 \%$ & $2 \%$ & $4 \%$ & $10 \%$ & $24 \%$ \\
\hline
\end{tabular}

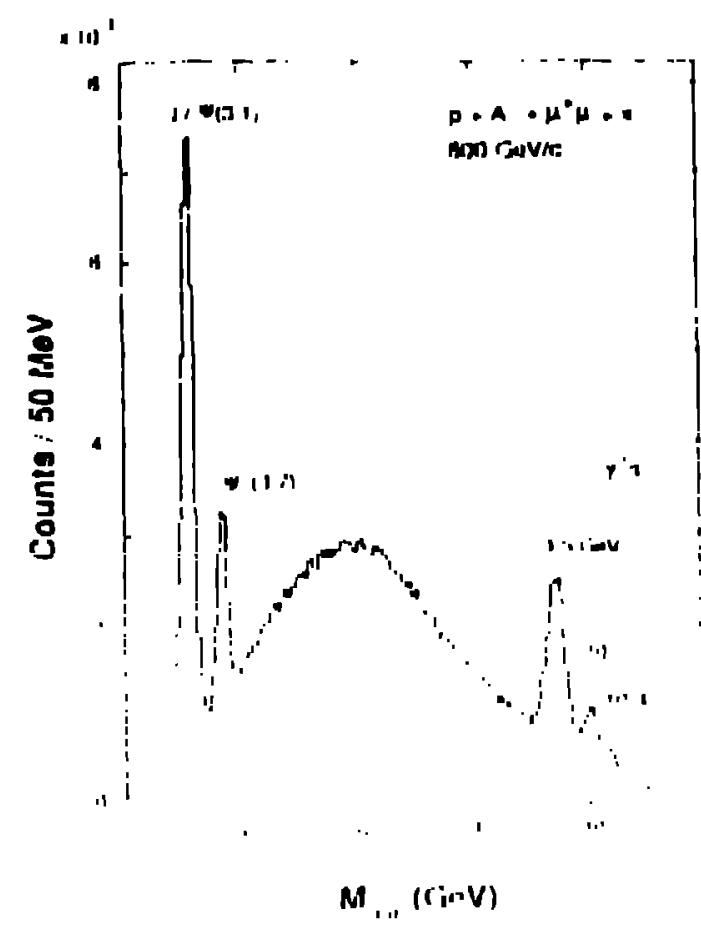

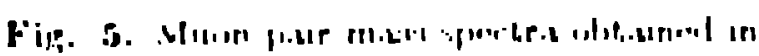

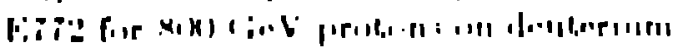

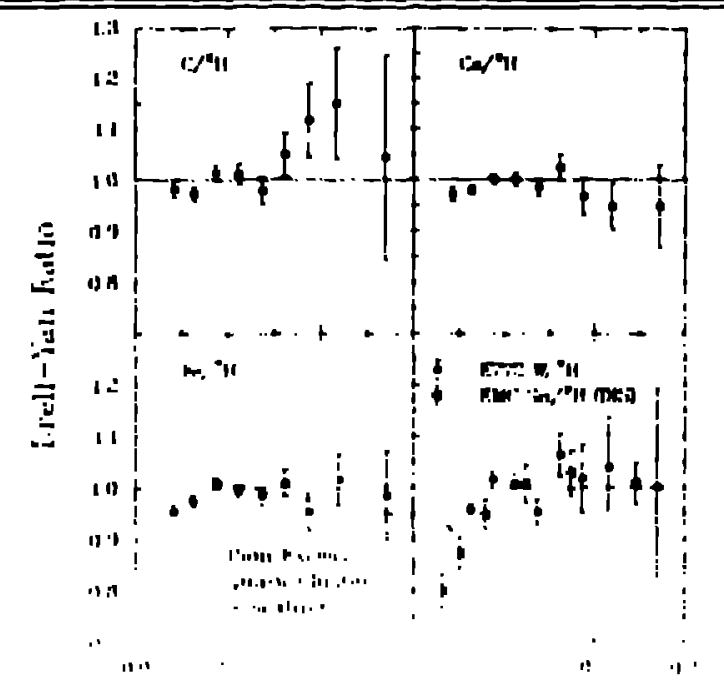

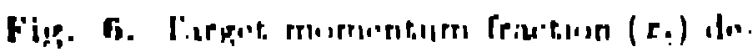

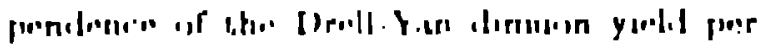

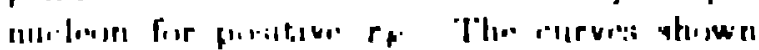

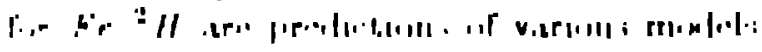

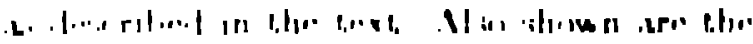

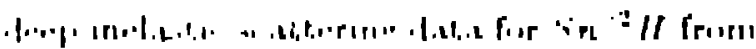

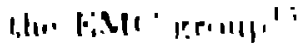


panel. The depletion in the shadowing region for the EMC data is apparently stronger than that seen in our data. However it is difficult to make a direct comparison since this EMC data in the shadowing region corresponds to $Q^{2}$ 's between 4 and $14 \mathrm{GeV}^{2}$ while the E772 data corresponds to $Q^{2}$ above $16 \mathrm{GeV}^{2}$. For our $\mathrm{Fe}^{2} \mathrm{H}$ ratio we compare with specific model calculations obtained from Hviang, Moss, and Peng. ${ }^{16}$ The pion excess model uses a $g_{0}^{\prime}=0.6$ and badly misses the data. The quark cluster calculation which follows the model of Carlson and Havens ${ }^{7}$ and uses a 6-quark cluster probability of $15 \%$ also badly misses the data. Only the rescaling model agrees at all with the data. The disagreement for $x \leq 0.1$ can presumably be fixed by adding in a depletion due to shadowing.

VI. A-dependence of Resonance Production

The mass dependence of the ratio of integrated cross sections (summed over $x_{f}$ and $p_{T}$ ) for resonance production from Ei72 is shown in Fig. 7 . Also shown for corr.parison is the Drell-Yan data whose total cross section has no mass dependence and lies on the horizontal line at $R=1$. All the resonances shown have a strong A-dependence with the lighter resonances $\left(J / \psi^{\prime}\right.$ and $\left.\psi^{\prime}\right)$ having the strongest suppression with mass. A simple fit of the form $R=A^{\prime \prime}$ has been done to the resonances (with the $J / \psi$ and $\psi^{\prime}$ fit together and the $\Upsilon$ 's fit together). The resulting $\alpha$ 's are 0.96 for the $\Upsilon$ and 0.92 for the $J / \psi$ and $\psi^{\prime}$. Although scme of this $\mathbf{A}$ dependence could be caused by a modification of the gluon structure function in nuclei it seems likely that most of it caused by nuclear effects in the final-state such as those described in Section III.

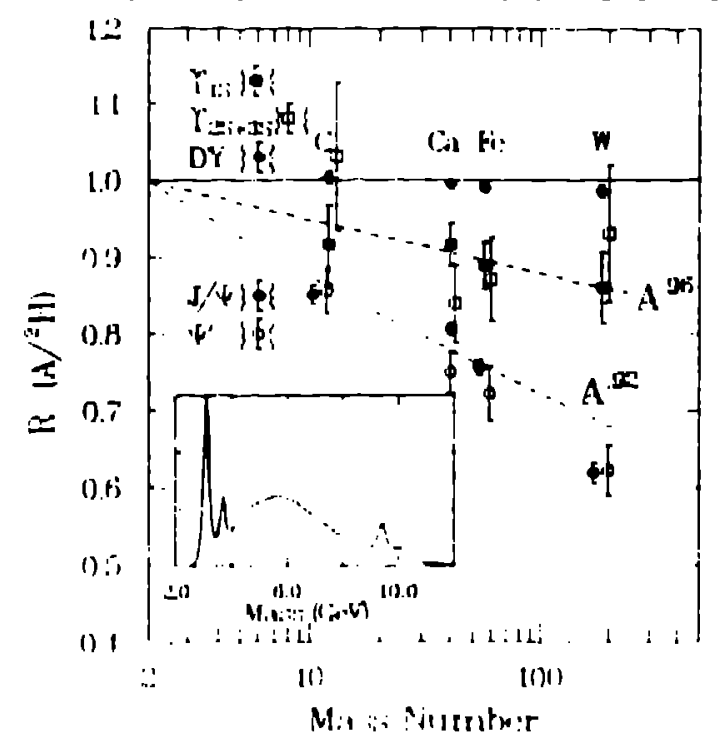

Fig. 7. Nidreleare-mitis dependence of rese-

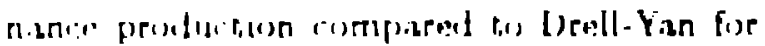

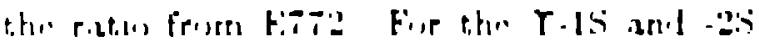

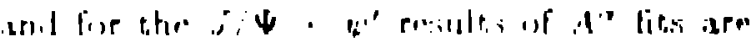
hilu n

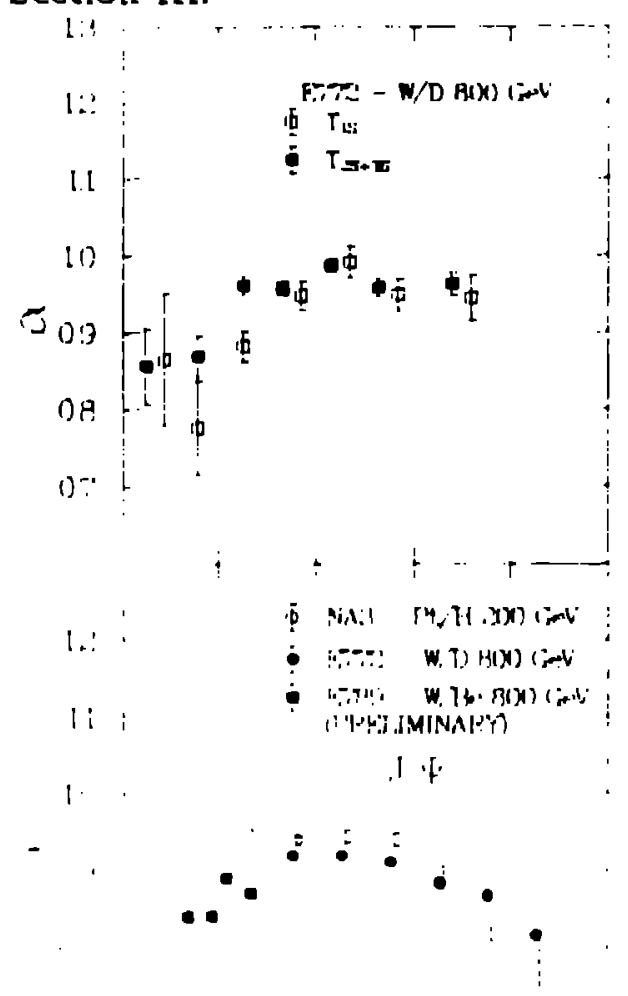

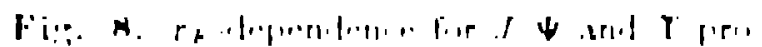

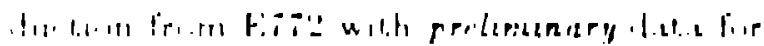

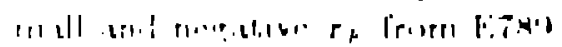

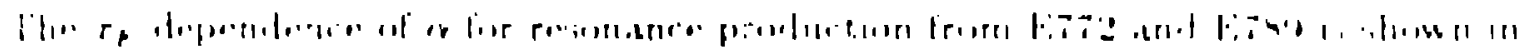

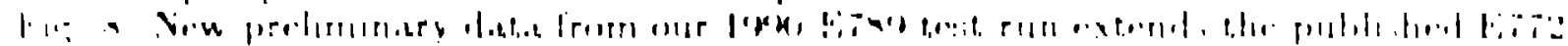

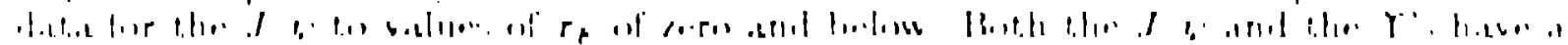


stronger suppreasion at $x_{\boldsymbol{r}} \simeq 0$ and below. This suppreasion, as suggested by Brcdsky and Vogt, 1 may be caused by the comovers which might be expected to have a larger overlap with a pre-meson when it is moving slower, as it would be in the small $x_{F}$ region. At large $x_{p}$ the $J / \psi$ shows an increasingly strong supp- ssion. Two different models claim to explain this effect. One is the intrinsic charm ...odel of Brodsly, which was mentioned earlier in this talk. The other is a simpler model from Gavin ${ }^{10}$ which explains this behavior as reaulting from energy loss effects in both the incident and final state. The basic idea behind this model can be understood in simple terms by considering the $I_{F}$ distribution of the production process which is depicted schematically in Fig. 9. When one goes from deterium to a heavy nucleus the additional energy loss can shift the effective $I_{F}$ distribution towards smaller $I_{r}$ resulting in a ratio which falls with larger $I_{f}$ as shown. A similar but smaller effect in the $D Y I_{F}$ dependence resulting from only the initial state is also explained within this model.
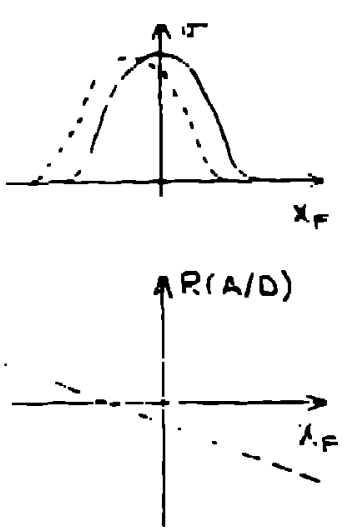

Fig. 9. Cartoon picture of how a shift in the Ir distribution affects the ratio.

Fig. 10. $p_{T}$-dependence for $J i \Psi$ and $T$ proiluition from Bitiz.

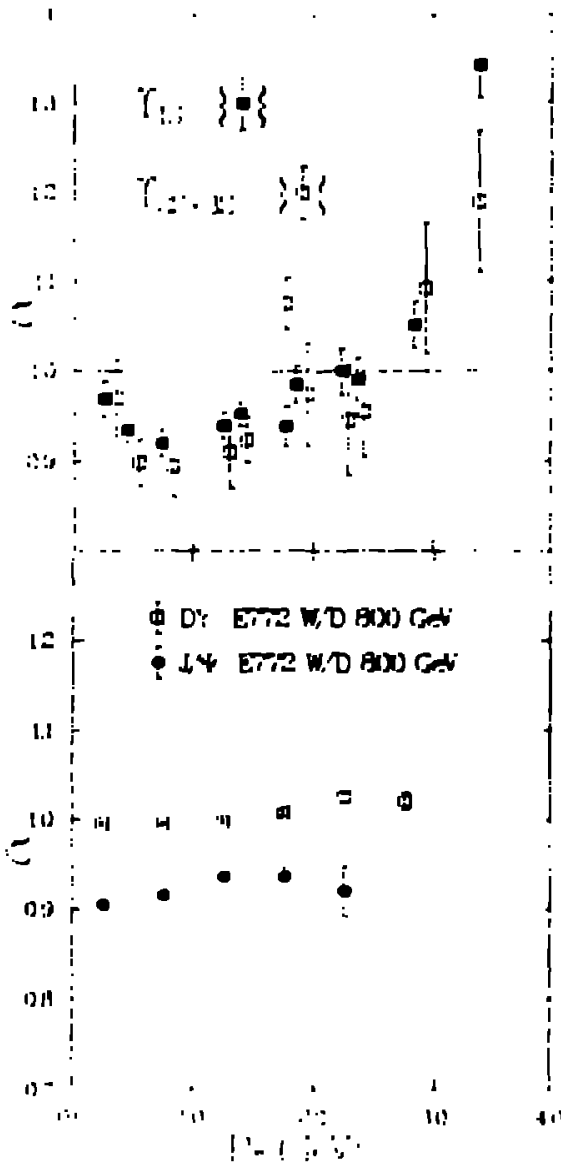

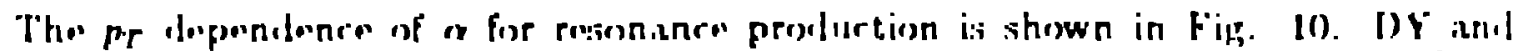

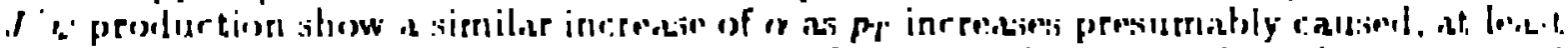

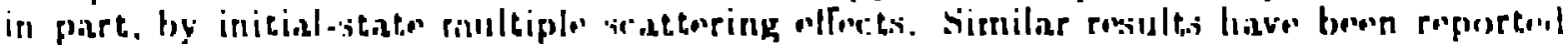

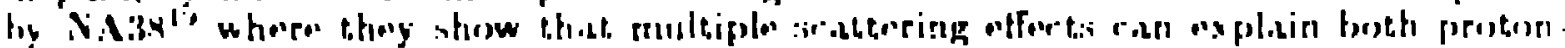

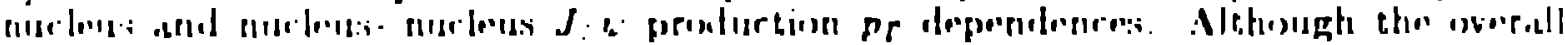

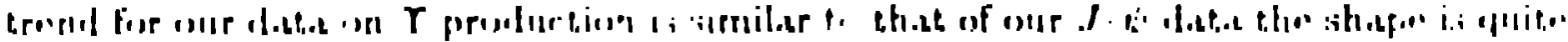

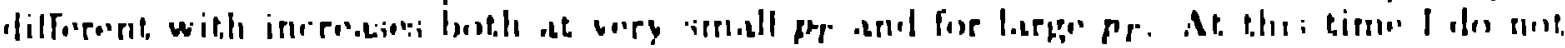

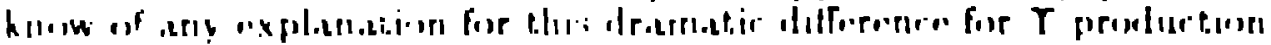

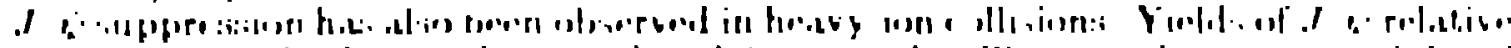

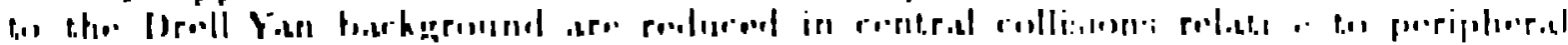

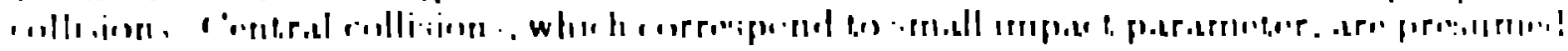


to be able to create quark-gluon plasma for which such a suppression was predicted. ${ }^{20}$ However it is clear that whatevei effects are responsible for the suppression of protonnucleus $J / \psi$ production may also play an important role in heavy-ion $J / \psi$ production and could explain most of the suppression seen with heavy-ions. Detailed comparisons have been done in Refs.. ${ }^{11,17}$

E789 is now running at Fermilab. One of the goals of this run is to determine the A-dependence of $D$ production and to compare to the results already obtained for $J / \psi$ and $I$ production. The standard mechanism for loss of $J / \psi$ 's due to interaction of a pre- $J / \psi$ with either the nucleus or comovers is dissociation into a $D-\bar{D}$ pair. In this case the loss of $J / \psi$ 's would apparently feed the production of $D$ 's, tending to enhance $D$ production. However recent data from WA82 for $D$-meson production suggests that the A-dependence for $D$ 's may be even stronger than that for the $J / \psi{ }^{21}$ If this is true ther. it may be inconsistant to describe the main mechanism for suppression of $J / \psi$ prodiction as dissociation to $D$ - $D$ pairs.

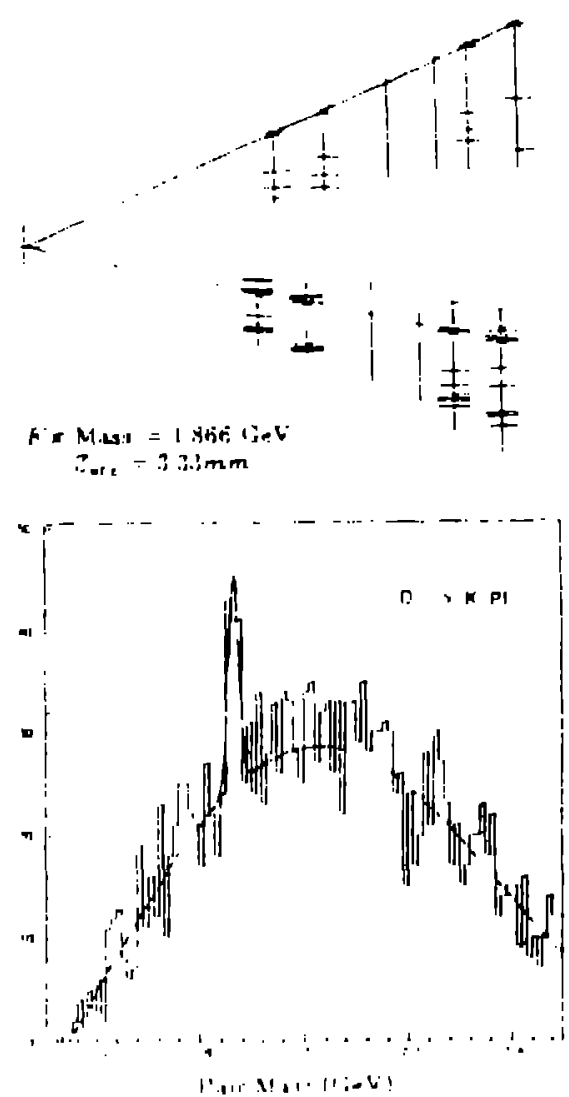

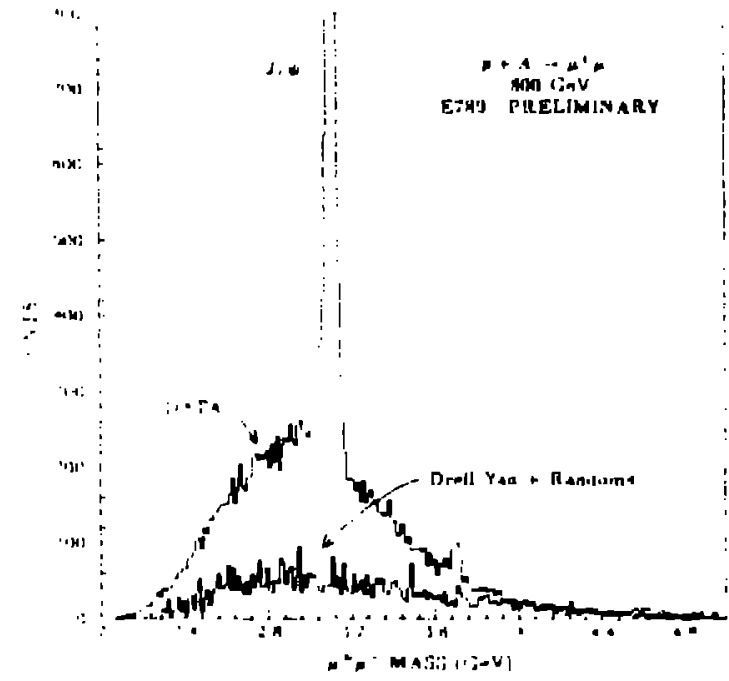

Fig. 12. The di-muon mass spectrum in the region of the $J / \Psi$ showing the predicted Drellyan $\div$ random contribution. The region below the $J / \Psi$ in mass is apparently dnminated by semi-leptonic decays of charm.

Fig. 11. A $D \rightarrow h \pi$ event seen in the 8-plane silieron vertex dietertor used durir.g our 1930 test run and the mass peak corresponding to downteream dercags of the D aliso from the text. run

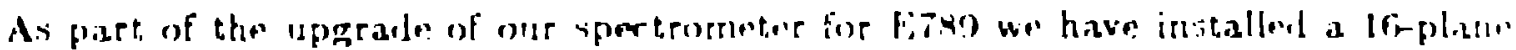

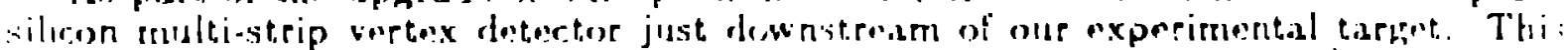

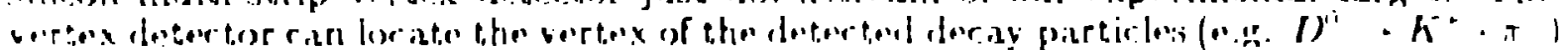

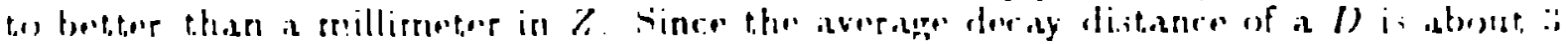

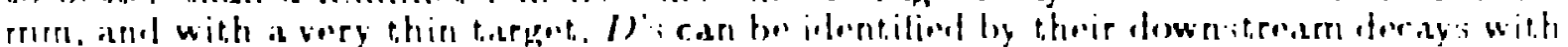

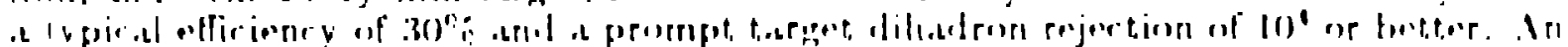

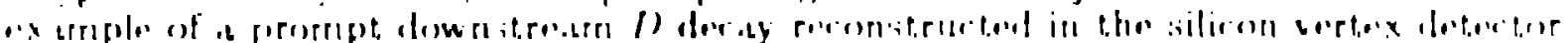

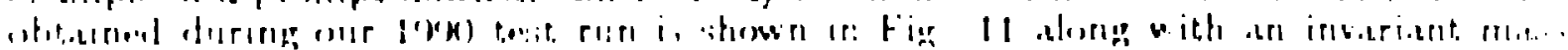

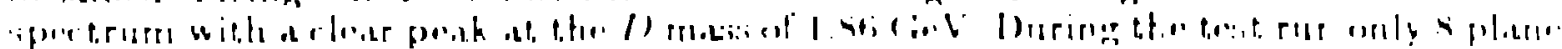

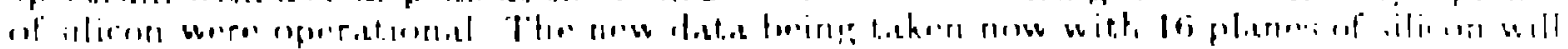


have correspondingly better efficiency for downstrearn decays and rejection of target backgrounds. Our estimate is that we already have approximately 7000 reconstuctable $D$ 's on tape (including efficiency losses) of which about 700 are from the $B e$ iarget and the rest from $W$. However it will take some time before we have our offline tracking tuned up adequately for the new silicon configuration and are able to produce new results including the $A$-dependence.

Another issue that our new data addresses is the content of the continuum in the mass region below the $J / \psi$. This is one of the regions where a signal from the quarkgluon plasm could be evident. Preliminary results from our recent measurements, as shown in Fig. 12, indicate that this region is domirated by char:... Calculations of the DY contribution, although somewhat unreliable in the mass region, show that the DY contribution is negligable compared to our data. The charm contribution, which comes from decay of a correlated $D-\bar{D}$ pair into di-lepto. 1 s via semi-leptonic decays, can be directly determined by measuring the $\mu^{ \pm} e=$ mass spectrum. We have done this and find that essentially all the continuum in the 2 to 5 GeV region can be explained by charm. The contribution from randoms in our experiment was found to be negligable. These conclusions are based on a very recent analysis of a very small portion of our data and will become more definitive as our analysis progresses. However it is already clear that signatures of quark-gluon plasma via continuum di-leptons in the 2 to $3 \mathrm{GeV}$ mass region will be overwhelmed by charm decays.

\section{Summary}

Precise measurements of the A-dependence of the Drell-Yan and of resonance $(J / \psi$, $\left.\psi^{\prime}, \Upsilon\right)$ production have been made in Fermilab E $7: 2$ and E 789 . The anti-quark structure function is not affected by the nurlear medium up to $x \leq 0.3$ except in the shadowing region at very small $I(I \leq 0.1)$. Models which produce a significant anti-quark enhancement or depletion for $0.1 \leq x \leq 0.3$ are ruled out. Vector meson production of $J / \psi, \psi^{\prime}$, and $\Upsilon$ all exhibit a large supression in a heavy nucleus with the strongest suppression for the $J / \psi$ and $\psi^{\prime \prime}$. This suppression is strongest for large $x_{F}$ and for $I_{F}$ at or below zero. The integrated A-dependence yields an $\alpha$ of .92 for the $J / \psi$ and $\psi^{\prime}$ and .96 for the $Y$ 's. It is not clear how to decouple structure and reaction mechanism effects in order to extract the A-dependence of the gluon structure function. The $p_{T}$ distribution is broadened for the $J / \psi$ presumably due to initial-state effects. For the $\Upsilon$ a more dramatic and complicated $p_{T}$ dependence is seer. ' ' which no simple explanation is known. $J / \psi$ suppression in proton-nucleus and in $: y$-ion collisions probably share common physical origins (e.g. initial and final-stace effects). Thus a clear understanding of proton-nucleus vector-meson production including more comprehensive data will be essnntial in order to understand production in heavy-ion collisions and to look for effects if a quark-gluon plasma. 


\section{References}

${ }^{1}$ M. Ericson and A. W. Thomas, Phys. Lett. 128 B (1983) 112.

${ }^{2}$ R. P. Bickerstaff, M. C. Birse, and G. A. Miller, Phys. Rev. Lett. 53 (1984) 2532.

${ }^{3}$ E. L. Berger, F. Coester, and R. B. Wiringa, Phys. Rev. D29 (1934) 398.

'F. E. Close, R. G. Roberts, and G. G. Ross, Ph;'s. Lett. 129B (1983) 346.

${ }^{5}$ R. L. Jaffee, Phys. Rev. Lett. 50 (1983) 228.

${ }^{6}$ F. E. Close, R. L. Jaffee, R. G. Roberts, and G. G. Ross, Phys. Rev. D31 (1985) 1004.

iC. E. Carlson and T. J. Havens, Phys. Rev. Lett. 51 (1983) 261.

${ }^{8}$ Hans J. Pirner and James P. Vary, Phys. Rev. Lett. 46 (1981) 1376.

${ }^{9}$ N. N. Nicolaev and V. I. Zakharov, Phys. Lett. B55 (1975) 397; A. H. Mueller and J. Qiu, Nucl. Phys. B268 (1986) 427; and Edmond L. Berger and Jianwei Qiu, Phys. Lett. B141 (1988) 119.

${ }^{10}$ Stanley J. Brodsky and Hung Jung Lu, Phys. Rev. Lett. 64 (1990) 1342.

"S. Gavin and R. Vogt, Nucl. Phys. B345 (1990) 104.

${ }^{12}$ Stanley J. Brodsky and Paul Hoyer, Phys. Rev. Lett. 63, (1989) 1566.

${ }^{13}$ D. M. Alde, et al., Phys. Rev. Lett. 64 (1990) 2479; Phys. Rev. Lett. 66 (1991) 133; and Phys. Rev. Lett. 66 (1991) 2285.

${ }^{14}$ G. Altarelli, R. K. Ellis, and G. Martinelli, Nucl. Phys. B157 (1979) 461; J. C. Collins, D. E. Soper, and G. Sterman, Phys. Lett. 134 B (1984) 263; and I. R. Kenyon, Rep. Prog. Phys. 45 (1982) 1261.

${ }^{15} \mathrm{~J}$. J. Aubert, et a.., Phys. Lett. B123 (1983) 275; and J. Ashman, et al., Phys. Lett. B202 (1988) 603.

${ }^{15}$ W. Y. P. Hwang, J. M. Moss, and J. C. Peng, Phys. Rev. D38 (1988) 2785.

${ }^{17}$ R. Vogt, S. J. Brodsky, and P. Hoyer, Nuel. Phys. B360 (1991) 67.; and S. J. Brodsky and $R$. Vogt, private communication.

"s. Gavin and J. Milana. William and Mary perprint WM-91-1LO.

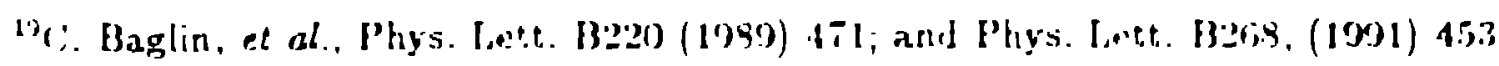

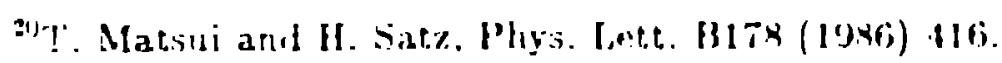

$=1$. Forian, ef al. (Wan2) preprint. 